\title{
A Phase II Randomized Dose Escalation Trial of Sorafenib in Patients With Advanced Hepatocellular Carcinoma
}

lorenza Rimassa, a ${ }^{2}$ Tiziana Pressiani, ${ }^{a}$ Corrado Boni, ${ }^{b}$ Carlo Carnaghi, ${ }^{a}$ Elena Rota Caremoli, ${ }^{c}$ Stefano Fagiuoli, ${ }^{d}$ Paolo Foa, ${ }^{e}$ Stefania Salvagni, ${ }^{f}$ Enrico Cortesi, ${ }^{g}$ Maria Chiara Tronconi, ${ }^{a}$ Nicola Personeni, ${ }^{a}$ Silvia Bozzarelli, ${ }^{a}$

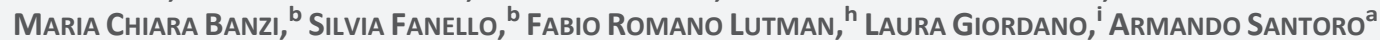

${ }^{a}$ Medical Oncology and Hematology Unit, Cancer Center, Humanitas Clinical and Research Center, Rozzano, Milan, Italy; ${ }^{b}$ Medical Oncology, Arcispedale Santa Maria Nuova, IRCCS, Reggio Emilia, Italy; ' Department of Oncology and Hematology, Ospedali Riuniti di Bergamo, Bergamo, Italy; ${ }^{d}$ Division of Gastroenterology and Transplant Hepatology, Department of Medicine, Ospedali Riuniti di Bergamo, Bergamo, Italy; ${ }^{e}$ Department of Oncology, San Paolo University Hospital, Milan, Italy; ${ }^{f}$ Oncology Division, Azienda Ospedaliero-Universitaria, Parma, Italy; ${ }^{8}$ Department of Radiology, Oncology and Human Pathology, Sapienza University of Rome,

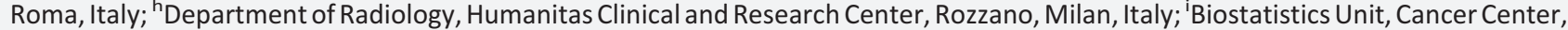
Humanitas Clinical and Research Center, Rozzano, Milan, Italy

Access the full results of this trial and read all published Clinical Trial Results at http://clinicaltrialresults.theoncologist.com/search/results

\section{Author Summary}

\section{ABSTRACT}

Background. Sorafenib has proven survival benefits in patients with advanced hepatocellular carcinoma (HCC). The viability of continuing sorafenib at a higher dosage in patients who experienced radiologic disease progression was investigated.

Methods. Patients who experienced disease progression while on sorafenib $400 \mathrm{mg}$ twice daily were randomized to sorafenib $600 \mathrm{mg}$ twice daily $(n=49)$ or best supportive care $(n=52)$. The primary end point was progression-free survival (PFS). Time to progression, overall survival, and safety were also evaluated.

Results. The study did not meet its primary end point. The difference in PFS between the sorafenib arm (3.91 months) and the best supportive care arm (2.69 months) did not reach statistical significance $(p=0.086)$. Adverse events were mainly grade 1-2 and similar across both groups. In the sorafenib arm, the most frequent events were diarrhea (80\%), weight loss (75\%), fatigue (67\%), hand-foot-skin reaction (49\%), abdominal pain (37\%), and stomatitis (26\%).

Conclusions. Escalated-dose sorafenib in patients with advanced HCC who progressed while on sorafenib, failed to provide any clinical benefit. Second-line treatment still remains an open issue to be explored in appropriate clinical trials. The Oncologist 2013;18:379-380

\section{DISCUSSION}

We report the results of a multicenter randomized phase II open-label study to evaluate the efficacy of sorafenib $600 \mathrm{mg}$ twice daily versus best supportive care (BSC) in patients with advanced hepatocellular carcinoma (HCC) progressing on sorafenib standard dose sorafenib. Pharmacokinetic data suggest that in patients with progressive disease, sorafenib dose escalation may restore adequate drug exposure and possibly antitumor activity.

ClinicalTrials.gov Identifier: NCT00490685 Sponsor: Humanitas Clinical and Research Center

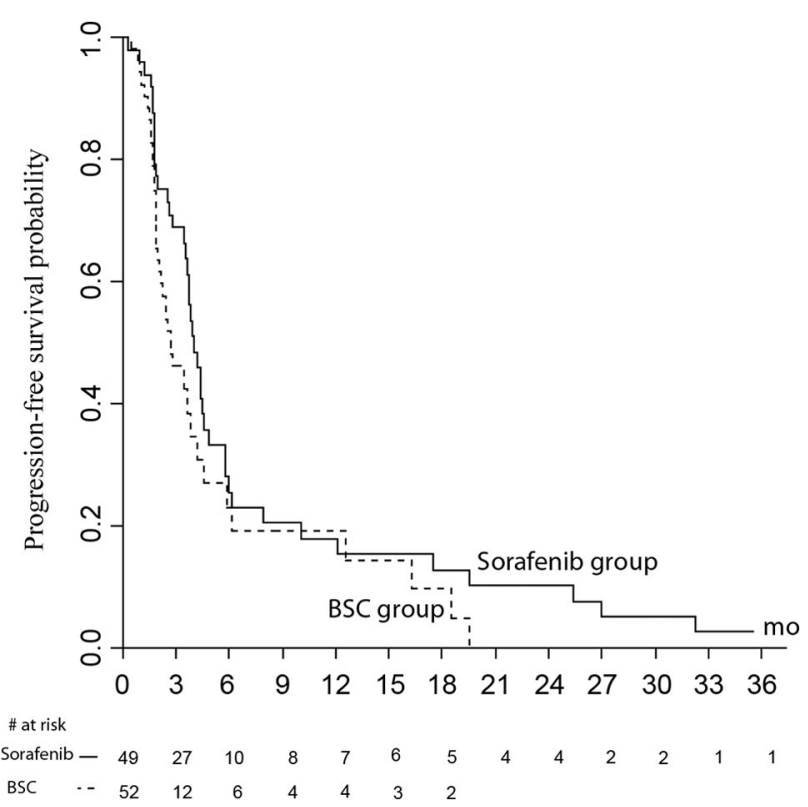

Figure 1. Probability of progression-free survival for sorafenib $(n=49)$ and BSC $(n=52)$ recipients.

Abbreviations: BSC, best supportive care; mo, months.

The study did not meet its primary and secondary end points of increasing progression-free survival (PFS) and overall survival (OS) when the dosage of sorafenib was increased to $600 \mathrm{mg}$ twice daily. The main result of this study is that sorafenib dose was increased effectively in three-quarters of patients, with $60 \%$ receiving $90 \%-100 \%$ of the protocol-specified dose. The randomized patients represent a highly selected population that tolerates sorafenib standard dose well without worsening of liver function or deterioration of clinical conditions.

Principal Investigator: Armando Santoro IRB Approved: Yes

Correspondence: Lorenza Rimassa, M.D., Medical Oncology and Hematology Unit, Cancer Center, Humanitas Clinical and Research Center, Via Manzoni 56, 20089 Rozzano, Milan, Italy. Telephone: + 39028224 4573; Fax: + 39028224 4590; E-Mail: lorenza.rimassa@cancercenter. humanitas.it; first published online in The Oncologist Express on April 11, 2013. CAlphaMed Press; the data published online to support this summary is the property of the authors. http://dx.doi.org/10.1634/theoncologist.2012-0221 
The most frequent adverse events (AEs) were diarrhea, weight loss, fatigue, hand-foot-skin reaction, abdominal pain, and stomatitis. Although AEs were common, the vast majority were grade $1-2$, suggesting that sorafenib dose escalation to $600 \mathrm{mg}$ twice daily is feasible and manageable.

This study had some potential limitations:

- Baseline characteristics were unbalanced, with more advanced disease and worse liver function (Child-Pugh class $B$ ) in the BSC arm;

- The open-label design determined that more patients in the sorafenib group underwent at least one postrandomization radiologic assessment;

- Response Evaluation Criteria in Solid Tumors (RECIST) was used rather than modified RECIST, which might provide more reliable assessment of treatment response;
- The twice-daily schedule might not be the best solution to increase sorafenib dosage-area under the curve was shown to increase infraproportionally with sorafenib doses higher than 400 mg twice daily; a fractionated schedule using three daily doses might be more efficient.

In conclusion, sorafenib $600 \mathrm{mg}$ twice daily is a viable and moderately tolerated treatment for a highly selected HCC population progressing on standard dose sorafenib. However, this study did not achieve its clinical endpoints, and secondline treatment in patients failing sorafenib still remains an open issue to be explored in clinical trials.

DisClOSURES

Author disclosures available online. 